\title{
Filmes nanométricos de titânia depositados via Magnetron Sputering DC
}

\author{
Nanometrics films of titaniun deposited via Magnetron Sputering DC
}

Suzy Pascoali ${ }^{1}$, Joel Brasil Borges ${ }^{1}$, Sérgio Artur Bianchini Bilac ${ }^{2}$, Orestes Estevam Alarcon ${ }^{2}$

\begin{abstract}
Resumo
Neste trabalho foram depositados filmes finos de $\mathrm{TiO}_{2}$ sobre vidro para modificar as características superficiais. Os filmes foram obtidos via Magnetron Sputtering em corrente contínua, uma técnica que forma de camadas de excelente qualidade, com controle de processo e reprodução da microestrutura. Foram analisadas a espessura e a morfologia. Os filmes nanométricos, com espessuras de 120 a $630 \mathrm{~nm}$, são ricos em nanocristais.
\end{abstract}

Palavras-chave: Titânia; Filmes finos; Magnetron Sputtering.

\begin{abstract}
In this work, $\mathrm{TiO}_{2}$ thin films were deposited in aim of modify the surface behavior to create autocleanness glass. Such films are obtained by Direct Current Magnetron Sputtering, this process can develop high quality films, because it has high control and microstructure reproducibility. Thickness and morphology were investigated. Nanometrics films were rich in nanocristals, thickness from 120 to $630 \mathrm{~nm}$.
\end{abstract}

Keywords: Titaniun; Thin films; Magnetron Sputtering. 


\section{Introdução}

Filmes finos de titânia são aplicados em produtos que desempenham função autolimpante. Esta propriedade é desenvolvida pelos filmes quando fotoirradiados por luz ultravioleta. $\mathrm{O}$ fóton pode liberar um elétron da banda de valência para a banda de condução. Este elétron ao chegar a superfície participa de reações que possibilitam a quebra de moléculas de hidrocarbonetos, até transformá-la apenas em água e gás carbônico, limpando a superfície de qualquer sujeira orgânica. Além disso, a titânia fotoirradiada pode absorver radicais hidroxilas, responsáveis pelo aumento de energia livre superficial, transformando a superfície em altamente hidrofílica, estes dois fenômenos em conjunto são chamados de efeito autolipante ${ }^{(1)}$.

Esta função pode ser apresentada por filmes que contenham nanocristais de anatásio ou rutilo ${ }^{(2)}$ e a rugosidade, espessura do filme podem amplificar a fotocatálise ${ }^{(3)}$.

A espessura do filme influencia na fotocatálise que melhora com aumento desta até um determinado patamar, no qual se mantém quase constante. $\mathrm{O}$ valor limite de fotocatálise é alcançado em filmes mais espessos ${ }^{(4)}$ devido a formação das partículas de $\mathrm{TiO}_{z}$ no interior do filme, diminuindo o número de locais ativos na superfície. $\mathrm{O}$ aumento da opacidade do filme e da difração da luz com a espessura diminui a passagem de luz através do filme e, consequentemente, a geração de par elétron-buraco.

Na maioria dos trabalhos em que é notada a variação da eficiência com a espessura, os filmes são submicromé$\operatorname{tricos}^{(5,6)}$. Nestes, a quebra de cadeias de hidrocarbonetos aumenta com a espessura. $\mathrm{O}$ aumento da eficiência deve-se provavelmente a dois fatores: o aumento da espessura proporciona um aumento da quantidade de cristais de dióxido de titânio disponíveis para participar da fotocatálise e o aumento do número de cristais eleva o número de par elétron-buraco gerado nos filmes de titânia ${ }^{(4)}$.

Um exemplo da influência causada pela rugosidade e pela estrutura cristalina é apresentado no trabalho de TAKEDA ${ }^{(3)}$, onde os filmes obtidos via sol gel, que apresentam menor quantidade de anatásio, tem a mesma eficiência de fotocatálise que os filmes obtidos via Sputtering. Isto mostra que os filmes obtidos via sol gel com a eficiência fotocatalítica equivalente aos obtidos via Sputtering se deve provavelmente a maior rugosidade deste (RMS). Uma vez que maiores rugosidade e porosidade do filme aumentam a sua superfície específica, facilitam o contato das substâncias adsorvidas com os cristais existentes, consequentemente aumentando a eficiência de fotocatálise.

A técnica de deposição por Magnetron Sputtering possibilita melhoria das características do filme e proporciona uma variedade de microestruturas e composição com os parâmetros de deposição.

Neste trabalho foram depositados via Magnetron Sputtering DC filmes com espessuras nanométricas de dióxido de titânio e foi analisada a morfologia superficial via Microscopia de Força Atômica.

\section{Experimental}

Filmes de espessura nanométrica foram obtidos via deposição reativa sobre placas de vidro plano comum em um reator Magnetron Sputtering DC industrial. As deposições foram realizadas na empresa APLIVAC, localizada na cidade de Campinas no Estado de São Paulo, utilizando parâmetros como: a tensão $(500 \mathrm{~V})$, corrente $(20$ - $30 \mathrm{~A})$, pressão $\left(10^{-2}\right.$ Torr ou $\left.1,3 \mathrm{~Pa}\right)$, pressão parcial $\left(100 \% \mathrm{O}_{2}\right)$.

$\mathrm{O}$ equipamento utilizado possui uma fonte de corrente contínua pulsada. A câmara de vácuo consiste em um cilindro de aço inoxidável, disposto na horizontal, com 600 mm de diâmetro e $600 \mathrm{~mm}$ de comprimento, as fotos são apresentadas nas Figs. 1a e $1 \mathrm{~b}$.

(a)

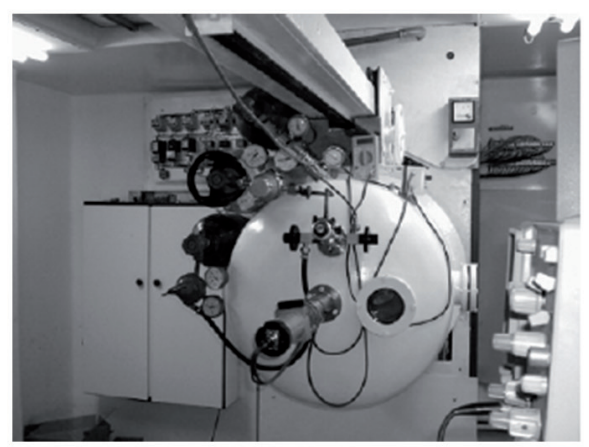

(b)

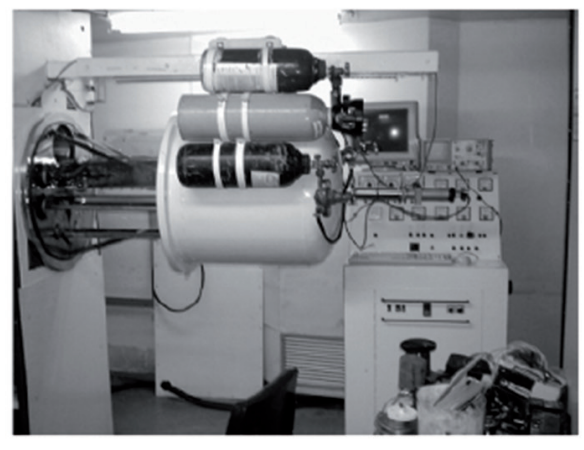

Figura 1. Sistema de deposição dos filmes via plasma via Magnetron Sputtering DC (a) vista frontal e (b) vista lateral. 
O sistema de vácuo consiste em uma bomba rotativa de $30 \mathrm{~m}^{3} / \mathrm{h}(8 \mathrm{l} / \mathrm{s})$, uma bomba Roots de $250 \mathrm{~m}^{3} / \mathrm{h}(70 \mathrm{l} / \mathrm{s})$ e uma difusora de 2000 1/s $\left(7200 \mathrm{~m}^{3} / \mathrm{h}\right)$. A deposição foi realizada por um Catodo Magnetron Planar de $500 \mathrm{~mm}$ de comprimento, equipado com uma fonte $D C$ pulsada que pode fornecer tensões de até $500 \mathrm{~V}$ e correntes de até $50 \mathrm{~A}$.

A resistência elétrica presente no porta substrato permitiu o aquecimento das amostras cerâmicas antes do início da deposição até a temperatura inicial de aproximadamente $220^{\circ} \mathrm{C}$. A deposição foi realizada a pressão de $10^{-2}$ Torr $(1,3 \mathrm{~Pa})$. O alvo utilizado foi de titânio de pureza 99,5\%, refrigerado por água em circuito fechado.

Para a formação do filme de dióxido de titânio foi utilizada uma atmosfera de descarga de oxigênio puro. Assim, o titânio da superfície do alvo reage com o oxigênio, oxidando e formando o composto de titânia para posterior Sputtering. Os átomos são arrancados da placa e viajam pelo plasma até o substrato. O processo de deposição realizado desta forma é denominado de Sputtering em modo óxido.

Este procedimento foi utilizado, pois o equipamento de Sputtering não tinha um espectrofotômetro de emissão óptica, ou qualquer outro dispositivo para detectar o envenenamento do alvo, e assim, possibilitar a manutenção dos parâmetros de deposição. Sem este dispositivo a deposição reativa se torna muito instável e de difícil controle.

Aliado a isto, Zeman ${ }^{(6)}$ demonstra que o aumento da pressão parcial favorece a eficiência da fotocatálise no filme de titânia. Deste modo optou-se em utilizar o modo óxido para a formação dos filmes de $\mathrm{TiO}_{2}$. Foram realizados três experimentos com tempos diferentes de deposição de 10, 15 e 60 minutos. Também foi variada a temperatura inicial do substrato no ensaio A. Uma vez que o reator pertence a uma indústria, foram realizados apenas três ensaios. Os parâmetros de deposição são mostrados na Tabela 1 .

A espessura das amostras foi definida através de espectrofotometria. Um espectrofotômetro de luz ultravioleta e visível foi utilizado para varrer o espectro de transmitância de placas planas de vidro, sendo transmitância tomada como a medida da fração da luz que atravessa um sólido. Neste equipamento um feixe de luz de comprimento de onda conhecido incide ortogonalmente sobre o filme depositado. Assim, faz-se a tomada da transmitância da placa para cada comprimento de onda. Como resultado tem-se um gráfico da transmitância em função do comprimento de onda.

A interferência do filme altera o número de franjas na curva da transmitância por comprimento de onda. Quanto maior o número de franjas, mais interferência e mais espesso é o filme. No gráfico da transmitância versus comprimento de onda gerado, se faz a contagem do número de franjas formadas num intervalo de comprimento de onda. Quanto maior o número de franjas, mais espesso é o filme ${ }^{(7)}$. A espessura pode ser calculada pela equação a seguir, onde $t$ é espessura do filme em picometros (pm) e $N$ é o número de franjas encontradas entre $\lambda_{1}$ e $\lambda_{2}$, que são os comprimentos de onda dados em nanometros (nm).

$$
t=\frac{N}{2} \lambda_{1} \cdot \lambda_{2}
$$

O estudo da morfologia da superfície do filme de titânia foi realizado por meio da técnica de microscopia de força atômica (AFM). O equipamento utilizado para a obtenção das imagens foi do tipo Picoscan da empresa Molecular Imaging e Scanner AF com área máxima de varredura de $6,2 \mu \mathrm{m}$ por $6,2 \mu \mathrm{m}$.

\section{Resultados e Discussões}

A Fig. 2 mostra a transmitância na região do visível e do ultravioleta dos filmes finos de titânia, depositados sobre lâminas de vidro comum. Os espectros de transmissão dos filmes depositados sobre o vidro apresentam característica bastante similar. O valor da transmitância dos vidros deste trabalho foi semelhante aquele desenvolvido pelos filmes comerciais apresentados anteriormente próximos a $95 \%$.

As lâminas em vidro utilizadas possuem alta absorção a comprimentos de ondas menores que $300 \mathrm{~nm}$, impossibilitando a análise da transmitância para valores de onda inferiores a este.

Tabela 1. Parâmetros de deposição.

\begin{tabular}{|c|c|c|c|c|c|}
\hline Vidro & Tensão [V] & Corrente [A] & Temp. Inicial $\left[{ }^{\circ} \mathrm{C}\right]$ & Pressão [Torr] & Temp. [minuto] \\
\hline $\mathrm{A}$ & 500 & 20 & 25 & $10^{-2}$ & 10 \\
\hline B & 500 & 20 & 220 & $10^{-2}$ & 15 \\
\hline $\mathrm{C}$ & 500 & 20 & 220 & $10^{-2}$ & 60 \\
\hline
\end{tabular}




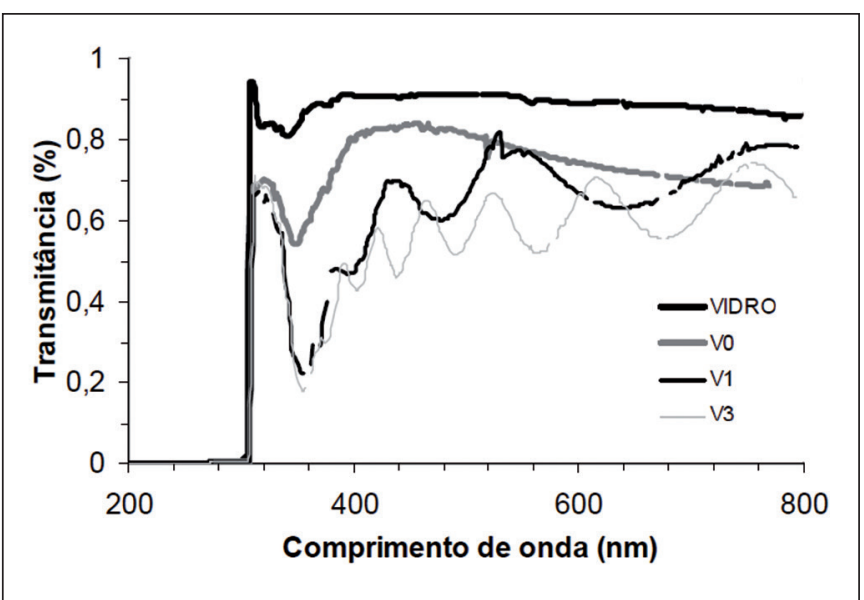

Figura 2. Transmitância na região do visível e do ultravioleta dos filmes finos de titânia depositados sobre vidro durante 10 minutos (A), durante 15 minutos (B), durante 60 minutos $(\mathrm{C})$.

Os vidros recobertos com $\mathrm{TiO}_{2}$ não tiveram sua transmitância significativamente diminuída. O filme depositado sobre vidro durante 10 minutos (Ensaio A) apresenta maior transmitância que os que foram depositados durante tempo maior de 15 e 60 minutos (Ensaio B e C).

A alta transparência adquirida possibilita o uso dos filmes mais finos em aplicação ótica. O desempenho na transmitância aqui encontrado é comparável ao desenvolvido por filmes ópticos na literatura obtidos via outros $\operatorname{processos}^{(8,9)}$.

Entre $\lambda$ de 308 e $320 \mathrm{~nm}$ ocorre um pico de transmitância de 70 \% com pequenas tremulações. Estas pequenas franjas na região UV podem ser devidas a uma transição eletrônica que ocorre em grupos hidroxilas $(\mathrm{OH})$ adsorvidos na superfície do vidro, advindas de reação com a água ${ }^{(10,11)}$.

Logo em seguida todos os filmes apresentaram um vale a cerca de $350 \mathrm{~nm}$, e suas transmitâncias foram diminuídas a $54 \%, 22 \%$ e $18 \%$, respectivamente nos ensaios de A, B e C. Após esta queda no valor da transmitância, a amostra A teve sua transmitância ligeiramente aumentada para $80 \%$ sofrendo um decréscimo gradativo até $68 \%$ em $760 \mathrm{~nm}$.
A transmitância é alta e quase constante com o comprimento de onda para filmes muito finos, mas diminui com o aumento da espessura do filme ${ }^{(12)}$. A transmitância das amostras B e C apresentam oscilações que variam em valores médios de 75 e $65 \%$, respectivamente.

A transmitância também pode ser útil para determinação da espessura de filmes finos. Onde o gráfico da transmitância é utilizado para a determinação do número de franjas. Nas curvas de transmitância, todos os espectros apresentaram uma franja entre 308 e aproximadamente $350 \mathrm{~nm}$, seguindo a tendência da transmitância apresentada pelo vidro comum. Para o cálculo da transmitância, este pico não foi considerado, por ser provavelmente uma interferência do vidro e não do filme.

Os filmes depositados durante 10, 15 e 60 minutos possuem 1, 3 e 6 franjas respectivamente. $O$ resultado da espessura calculada versus a transmitância depende do número de franjas. Quanto mais franja, maior a espessura do filme.

A espessura calculada pela transmitância foi de 120 , 295 e $630 \mathrm{~nm}$, respectivamente, para o filme depositado em 10, 15 e 60 minutos. Na medida realizada nos filmes via Microsocopia Eletrônica de Varredura MEV, as espessuras médias foram de 290 e $560 \mathrm{~nm}$, respectivamente para o filme depositado durante 15 e 60 minutos. O resultado da espessura obtida via transmitância é mostrado na Tabela 2.

A medida no MEV do filme de $\mathrm{TiO}_{2}$ foi comparada com a quantificação da espessura do mesmo, via transmitância do filme depositado sobre placa de vidro comum. Há uma semelhança grande entre os resultados obtidos via espectrometria e pela medida da secção transversal do filme obtida via MEV. Assim, se confirma como esperado que as técnicas de espectrometria de transmitância e a micrografia do MEV como formas apropriadas para obtenção da espessura de filmes nanométricos.

Os resultados de análise de Difratometria de Raio-X mostram que os filmes depositados por 60 minutos apresentam picos relativos predominantemente a fase anatásio,

Tabela 2. Resultado da espessura calculada por transmitância nos filmes de $\mathrm{TiO}_{2}$ depositados via Magnetron Sputtering DC sobre vidro comum.

\begin{tabular}{|c|c|c|c|c|}
\hline Filme & $\mathbf{N}$ & $\lambda_{1}(\mathrm{~nm})$ & $\lambda_{2}(\mathrm{~nm})$ & Transmitância $t(\mathrm{~nm})$ \\
\hline A & 1 & 308 & 784 & 120 \\
\hline B & 3 & 308 & 640 & 295 \\
\hline $\mathrm{C}$ & 6 & 308 & 680 & 630 \\
\hline
\end{tabular}


o depositado por 15 minutos apresenta picos de ambas as fases, tanto de rutilo como anatásio, não foi possível verificar a presença de picos no difratograma da amostra depositada por $10 \mathrm{~min}$ (Pascoali, 2006).

A morfologia do filme foi analisada via microscopia de força atômica (AFM). A superfície da amostra foi varrida por um cantilever, para a obtenção das características morfológicas do filme de titânia.

Na Fig. 3 é apresentada a micrografia da superfície do vidro comum, sem filme. $\mathrm{O}$ vidro apresenta pequenos círculos na morfologia da sua superfície. Estas protuberâncias no relevo apresentam altura na ordem de décimos de Ångström, demonstrando que o vidro utilizado possui uma superfície muito lisa.

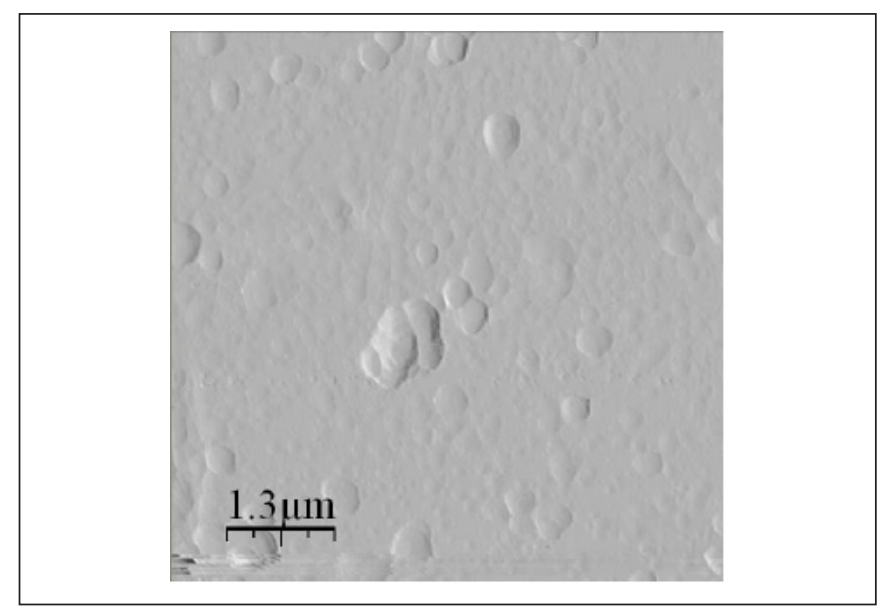

Figura 3. Resultados da microscopia de força atômica realizada no vidro comum. Imagem $2 \mathrm{D}$ da superfície do vidro com uma área de $6,3 \mu \mathrm{m}$ por $6,3 \mu \mathrm{m}$.

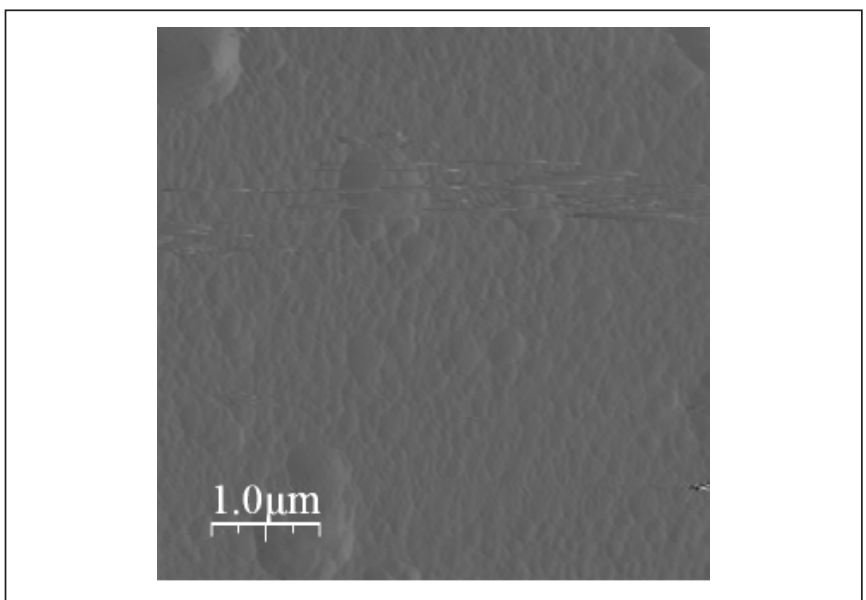

Figura 4. Resultados da microscopia de força atômica realizada na amostra $B$, com filme de titânia depositado durante 15 minutos. Imagem 2D da superfície de B com uma área de $5 \mu \mathrm{m}$ por $5 \mu \mathrm{m}$. Rugosidade na ordem de unidade de nanometro.
As imagens da superfície dos filmes de titânia depositados sobre vidro são apresentadas nas Figs. 4 e 5 . O relevo das amostras com filme apresenta rugosidade na ordem de unidade de nanômetro. A diferença de rugosidade é maior mais de cem vezes entre a superfície do vidro e a superfície dos filmes depositados sobre o vidro. Um indicativo de que a rugosidade destas amostras se deve a presença do filme, ou seja, que o relevo lido no AFM corresponde, em grande parte, ao relevo do filme uma vez que o vidro é muito liso.

As micrografias em AFM dos filmes de titânia obtidos via Magnetron Sputtering DC apresentam uma superfície contínua e formada por grãos. Estas estruturas na verdade representam o topo das colunas dos filmes e são constituídas por um aglomerado de nano-cristais.

O tamanho destes grãos é diferente entre os filmes. A superfície da amostra de vidro com filme depositado durante 15 minutos consiste de pequenos polígonos de, aproximadamente, $70 \mathrm{~nm}$ de diâmetro. O mesmo substrato, filme depositado durante 60 minutos apresenta uma topografia formada por grãos maiores, aproximadamente $100 \mathrm{~nm}$. Verificação é compatível com a literatura, pois características semelhantes foram obtidas nos filmes do trabalho de Zeman, Chaiyakun ${ }^{(13,14)}$.

A mudança na morfologia entre os filmes $\mathrm{B}$ e $\mathrm{C}$ foi provocada pelo aumento do tempo de deposição. Os filmes ficam mais espessos e os grãos maiores com o aumento do tempo de deposição.

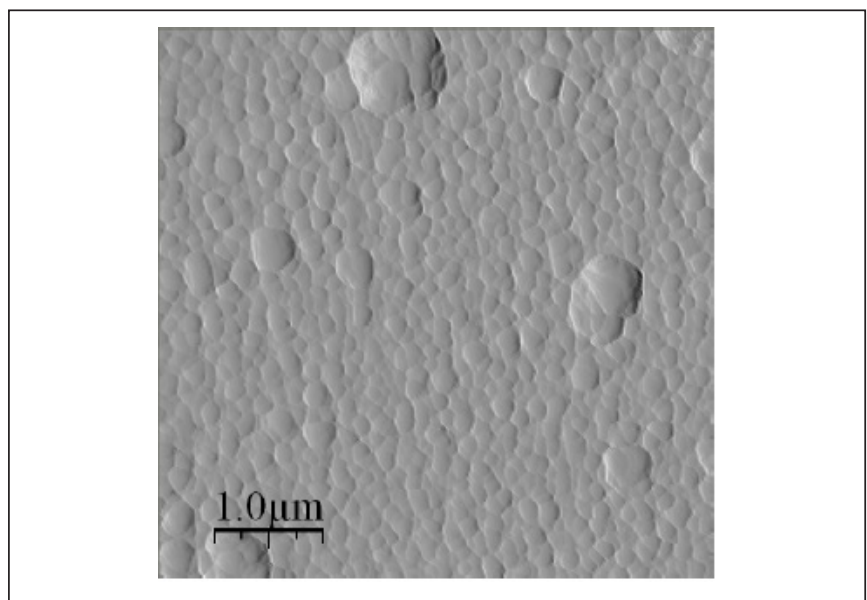

Figura 5. Resultados da microscopia de força atômica realizada na amostra $\mathrm{C}$, com filme de titânia depositado durante 60 minutos. Imagem 2D da superfície de C com uma área de $5 \mu \mathrm{m}$ por $5 \mu \mathrm{m}$. Rugosidade na ordem de unidade de nanometro. 


\section{Conclusões}

Filmes nanométricos de titânia foram depositados via Magnetron Sputtering DC em escala industrial.

Os parâmetros de deposição modificaram a morfologia dos filmes, os depositados durante um tempo menor apresentaram estrutura granular mais fina, que foi mais eficiente na fotocatálise.

\section{Referências}

1. Fujishima, A.; Rao, T.N.; Tryk, D. A.; Titanium dioxide photocatalysis. Journal of Photochemistry and Photobiology C: Photochemistry reviews, v. 1, p. 1-21, 2000.

2. Watanabe, T.; Nakajima, A; Wang, G R.; Minabe, M.; Koizumi, S.; Fujishima, A.; Hashimoto, K.; Photocatalytic activity and photoinduced hydrophilicity of titanium dioxide coated glass, Thin Solid Films, v 351, p 260-263, 1999.

3. Takeda, S.; Suzuki, S.; Odaka, H.; Hosono, H.; Photocatalytic $\mathrm{TiO}_{2}$ thin film deposited onto glass by DC Magnetron Sputtering. Thin solid films 392 (2001) 338-344. HABIBI. M. H.;

4. Habibi, M. H.; Talebian, N.; Choi, J. H.; The effect of annealing on photocatalytic properties of nanostructured titanium dioxide thin films. Dyes and Pigments 73 (2007) 103 e 110.

5. Chang, J. T.; Su, C.W; He, J.L.; Photocatalytic $\mathrm{TiO}_{2}$ film prepared using arc ion plating. Surface \& Coatings Technology 200 (2006) 3027-3034.

6. Zeman, P.; Takabayashi, S.; Nano-scaled photocatalytic $\mathrm{TiO}_{2}$ thin films prepared 2 by Magnetron Sputtering. Thin Solid Films 433 (2003) 57-62

7. Cardoso,W. S.; Longo, C.; Paoli, M. A.; Preparação de eletrodos opticamente transparentes. Quim. Nova, Vol. 28, No. 2, 345-349, 2005.

8. Chuang, H.; Chen, D.; Catalyst-free low temperature synthesis of discrete anatase titanium dioxide nanocrystals

\section{Agradecimentos}

À empresa APLIVAC, pela gentileza em ceder o equipamento de Magnetron Sputtering, em especial ao Sérgio Birolli; ao Prof. André Pasa do Departamento de Física da UFSC, ao Max Munford e Ricardo Zandonay, pelos ensaios no AFM; o Prof. Danilo Wilhelm Filho do Departamento de Ecologia e Zoologia ECZ/UFSC, pela gentileza em ceder o equipamento de espectrometria e à CAPES e PGMAT, pela bolsa de estudo concedida.

with highly thermal stability and UVC-cut capability. J Nanopart Res (2008) 10:233-241.

9. Wang, J.; et al. Heat Treatment of nanometer anatase powder and its photocatalytic activity for degradation of acid red B dye under visible light irradiation. Inorganic Materials, 2008, Vol. 44, No. 6, pp. 608-614.

10. Takeda. S.; Fukuwa, M.; Surface OH groups governing surface chemical properties of $\mathrm{SiO}_{2}$ thin films deposited by $\mathrm{RF}$ Magnetron Sputtering. Thin Solid Films 444 (2003) 153-157.

11. Xavier, L. F. W.; Moreira, I. M..N. S.; Higarashi, M. M.; Moreira, J.C.; Ferreira, L.F.V.; Oliveira, A.S.; Photodegradation of polycyclic aromatic hydrocarbons (PAHs) adsorbed onto silica gel chromatographic plates impregnated with $\mathrm{TiO}_{2}$. Quím. Nova, May/June 2005, vol.28, no.3, p.409-413. ISSN 0100-4042.

12. Teixeira, J. D.; Desenvolvimento e caracterização de filmes de materiais fracamente condutores depositados por magnetron sputtering para uso em isoladores elétricos de porcelana. Florianópolis, 2007. 89 f. Dissertação (Mestrado) - Universidade Federal de Santa Catarina, Centro Tecnológico. Programa de Pós-Graduação em Ciência e Engenharia de Materiais.

13. Zeman, P.; Takabayashi, S.; Surface and Coatings Technology 153 (2002) 93-99

14. Chaiyakun, S.;Pokaipisit,A.;Limsuwan,P.; Ngotawornchai, B.; Growth and characterization of nanostructured anatase phase $\mathrm{TiO}_{2}$ thin films prepared by DC reactive unbalanced magnetron sputtering. Appl Phys A (2009) 95: 579-587 